\title{
SIEDLUNGSFORMENKARTE DER SLOWAKEI
}

\author{
Von Stefan Fekete
}

Mit 2 Karten

Der Slowakei, dem östlichen Teile der Tschechoslowakei, wurde bisher vom siedlungsgeographischen Gesichtspunkt aus nur sehr geringe Aufmerksamkeit gewidmet. Mit dem systematischen Studium der ländlichen Siedlungen der Slowakei hat sich bisher niemand befaßt. Immerhin berücksichtigten die Geographen die Siedlungen entweder in ihren Studien der regionalen Geographie, so H. Hassinger, J. Hromadka, F. Machatschek, Vl. Kubrjovyc, oder in selbständigen Beiträgen, wie A. Malaschofsky, J. Kral, J. Hromadka, J. Martinka, St. Jansak usw. Als erster befaßt sich der Autor mit der systematischen Erforschung der ländlichen Siedlungen der Slowakei auf Grund der einschlägigen Literatur, kollektiver Arbeit seiner Hörer wie auch eigenen Studiums im Archiv der Katastralkarten in Bratislava und unmittelbar im Terrain. Die Ergebnisse seiner Arbeit legte er in seiner Schrift "Typen der ländlichen Siedlungen in der Slowakei», nieder, die von der Slowakischen Geographischen Gesellschaft in Bratislava 1947 herausgegeben wurde 1 . Ihr liegt die hier erstmals publizierte Siedlungsformenkarte der Slowakei zugrunde, die im folgenden kurz kommentiert sei.

Als Grundlage dienten mir Katastralkarten 1:2880 und andere ältere und neuere Karten, so daß es möglich war, auch die enge Beziehung zwischen Grundriß, Ackerland und ganzem Kataster zu studieren. Ich fertigte die Siedlungsformenkarte nach der Markierungsmethode (Zeichenmethode) im Maßstab 1:400000 an und hielt mich dabei an folgende Grundsätze: Wenn in der Natur zwei oder mehr ländliche Siedlungen so weit verwachsen sind, daß sie ein geographisches Ganzes bilden, dann sind sie auf der Karte nur durch eine Grundrißmarke (ein Grundrißzeichen) bezeichnet. Wenn in einem Kataster mehrere ländliche Siedlungen vorkommen, ist jede auf der Karte durch eine besondere Grundrißmarke bezeichnet. In den Streusiedlungsgebieten, wo außer zerstreuten Häusern auch ein konzentrierter Siedlungskern besteht, wird dieser Kern durch die betreffende Grundrißmarke (Grundrißzeichen) bezeichnet und außerdem die Fläche des Katasters punktiert. Einzelhöfe sind auf der Karte je nach der Größe durch drei oder vier Punkte dargestellt.

Bei der Klassifizierung der ländlichen Siedlungen benützte ich die genetische Methode, mit Ausnahme der Haufendörfer. Aber auch bei letzteren wird diese Methode nicht ganz ausgeschlossen. Die genetische Darstellung von Haufendörfern würde ihre Typisierung nach dem Grundriß ihres Kerns erfordern. Bei der Mehrzahl der Haufendörfer überwiegt jedoch der außerhalb des Kerns liegende Teil, so daß sich der Kern im Gesamtgrundriß beinahe verliert. Ein solches Dorf nach seinem Kern zu typisieren, könnte deshalb bedeuten, daß ein längst vergangener Zustand typologisch rekonstruiert würde. Deshalb reicht bei der Typisierung der Haufendörfer die rein genetische Methode nicht aus.

Unter dem Typ des Haufendorfes fasse ich den Untertyp des regelmäßigen und unregelmäßigen Haufendorfes zusammen. Der Untertyp des regelmäßigen Haufendorfes entstand gewöhnlich aus Dörfern anderen Typs durch Aufschwung im Anwachsen; der des unregelmäßigen Haufendorfes formte sich sogleich von Anfang an als Haufendorf. In genetischer Auffassung ist der Untertyp des unregelmäßigen ein wirkliches Haufendorf, während der Untertyp des regelmäßigen Haufendorfes nach der Grundrißform der Kerns und nach seiner Entwicklung in verschiedene Grundrißtypen ländlicher Siedlungen einbezogen werden kann.

1 S. Fekete: Typy viedieckeho osidlenia na Slovenska. Bratislava 1947. 


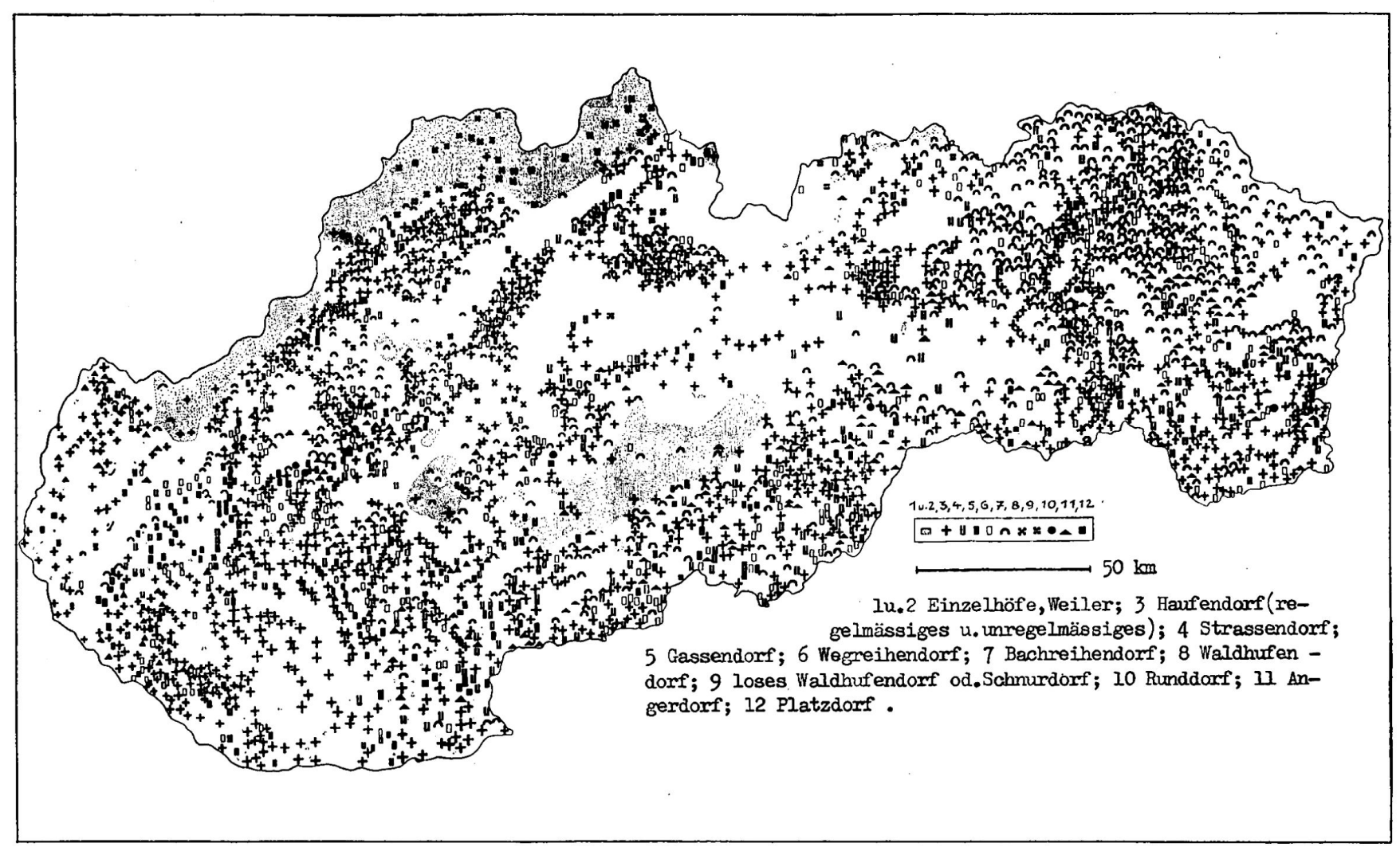

Karte 1: Siedlungsformen der Slowakei 
Um die Grundriß- und damit auch die gesamte Siedlungscharakteristik der ländlichen Siedlungen richtig festhalten zu können, bezeichnete ich beide Untertypen von Haufendörfern mit einem gemeinsamen Zeichen, obgleich ich mir der verschiedenartigen Entwicklung bewußt bin.

Im Einklang mit der genetischen Methode war es jedoch nötig, einige Korrekturen durchzuführen, damit die Karte gewisse Tatsachen nicht entstelle, die durch die Entwicklung bedingt sind. Diese Korrekturen hält die Karte 2 fest, auf der nur die unregelmäßigen Haufendörfer eingezeichnet sind. Nur diese werden nämlich genetisch als echte Haufendörfer angesehen. Der Vergleich der Karte 1 mit 2 zeigt sodann,

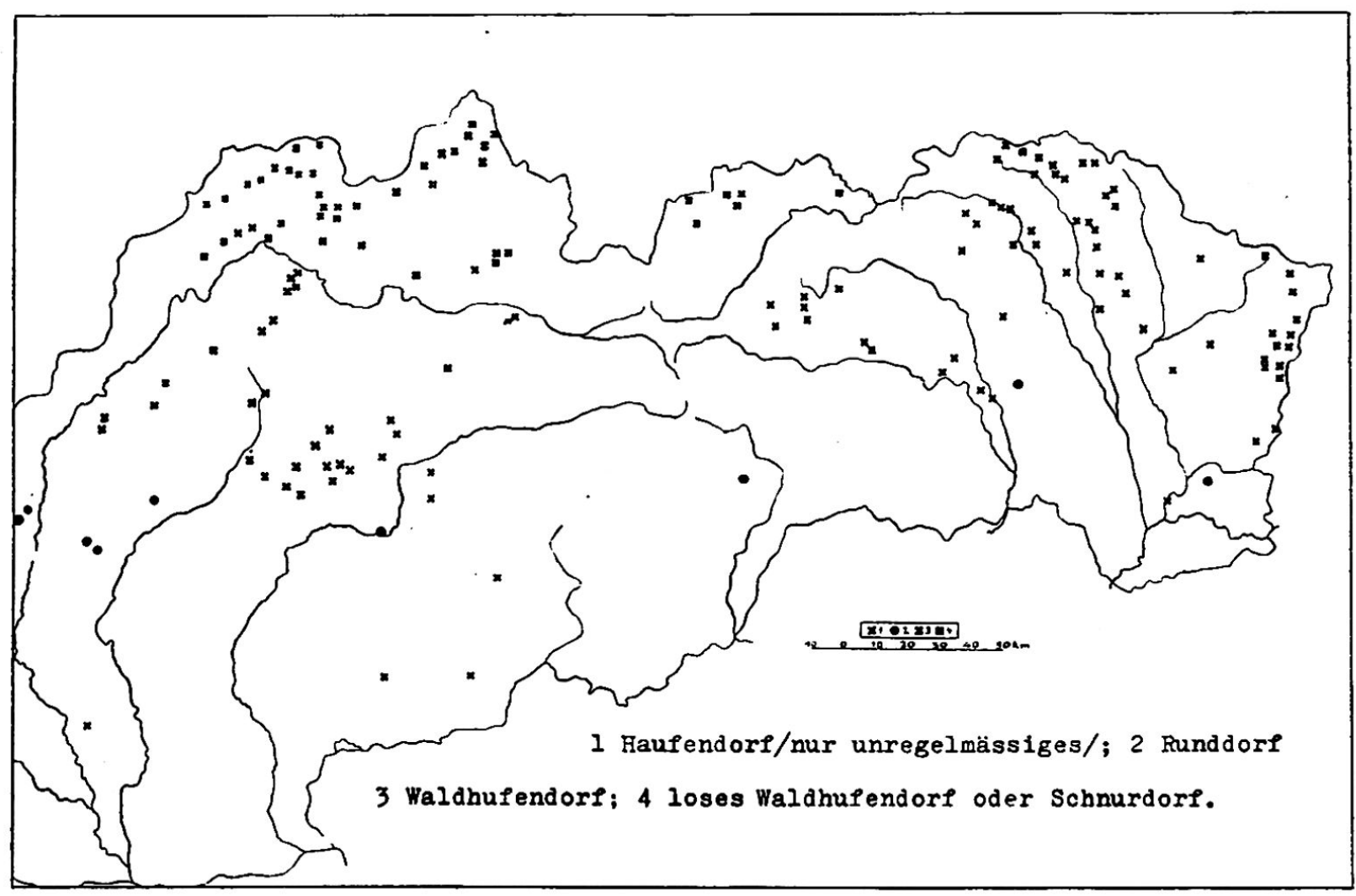

Karte 2: Einige Typen slowakischer Siedlungsformen

$\mathrm{da} ß$ fast alle Haufendörfer, außer jenen, die auf der Karte 2 eingezeichnet sind, ursprünglich Dörfer anderen Typs sind, wobei sich ihr ehemaliger Typ in ihrem heutigen Kern mit größerer oder geringerer Genauigkeit erhalten hat. Aus den beigefügten Karten läßt sich Wesentliches über den Wuchs und die Ausdehnung der Haufendörfer herauslesen. Es ist aus ihnen ersichtlich, daß viele Dörfer, die ursprünglich anderen Typen angehörten, erst später infolge der Entwicklung des Grundrisses zu Haufendörfern wurden und $\mathrm{da} \beta$ diese Veränderung von Dörfern anderen Typs in Haufendörfer am intensivsten in Talkesseln und Niederungen, weniger in den Gebirgen vor sich ging, da vor allem in den Niederungen und weiter auch in den Talkesseln genügend Raum für das Anwachsen des Grundrisses in jeder beliebigen Richtung vorhanden ist. Ferner wurden Ebenen und Talbecken zuerst besiedelt, so daß die in diesen liegenden Dörfer im Hinblick auf den Grundriß am meisten entwickelt sind.

Karte 1 zeigt die Verbreitung der einzelnen Siedlungsformen. Wenn sich auch in Einzelheiten bedeutende Abweichungen ergeben, ist daraus dennoch folgendes festzustellen: Einzelhöfe und Weiler sind an Hügelland und Gebirge gebunden. Sehr wenige davon liegen in Talkesseln und Niederungen. Haufendörfer sind Siedlungen der Tal- 
kessel und Ebenen. Sie bilden hier ungefähr $50 \%$ aller Dörfer. In geringerem Maßstab kommen sie auch in Gebirgen vor, etwa $30 \%$. Straßen-, Gassen- und Wegreihendörfer sind am zahlreichsten in Niederungen, dann in Talkesseln und schließlich im Gebirge. Bachreihendörfer sind in Niederungen am seltensten, häufiger in Talkesseln, und am meisten sind sie in Gebirgen anzutreffen.

Waldhufendörfer finden wir in den Niederungen nicht, da dieser Typ erst zu einer Zeit zu uns drang, als in den Niederungen kein Raum mehr dafür war. Es blieben für diesen Typ nur Talkessel und die Ränder der Gebirge. Lose Waldhufendörfer als Normtypen der walachisch-goralischen Kolonisation beschränken sich auf die Flyschzone der Karpaten. Selten finden sie sich in Talkesseln, niemals jedoch in Niederungen.

Bei den Rund-, Anger- und Platzdörfern sind keine besonderen Beziehungen zu den Erdoberflächenformen zu beobachten, da sie in annähernd gleich hohem Prozentverhältnis in Niederungen, Talbecken und Gebirgen vorkommen. Auf ihre Ausdehnung nahmen eher Eingriffe der menschlichen Hand als Naturfaktoren Einfluß.

\section{CARTE DES TYPES D'HABITATIONS DE LA SLOWAQUIE}

L'auteur présente une carte des types d'habitations, basée premièrement sur une recherche approfondie et systématique du pays entier.

\section{CARTA DEI TIPI DI INSEDIAMENTO DELLA SLOVACCHIA}

L'autore presenta una carta dei tipi di insediamento della Slovacchia basandosi sugli primi studi sistematici e complessivi del genere in questo paese.

\section{MITTEILUNGEN DER EIDG. LANDESTOPOGRAPHIE ${ }^{1}$}

\section{Von KarL SchNeider}

I. Farbenphotographische Landschafts- und Kartenbilder. Für Anwendungen der Farbenphotographie bei der Eidgenössischen Landestopographie ergeben sich hauptsächlich zwei besonders wichtige und wertvolle Möglichkeiten. Es sind dies einerseits die naturlandschaftliche Dokumentation des zu topographierenden und kartierenden Geländes mittels farbenphotographischer Fliegeraufnahmen, anderseits die farbenphotographische Vervielfältigung von mehrfarbigen Plan- und Kartenoriginalen. Hierüber hat die Eidgenössische Landestopographie Untersuchungen angestellt und praktische Versuche unternommen.

Es liegen erstmalige und vorläufige Ergebnisse vor, die meines Erachtens für Naturwissenschafter von allgemeinem und für Geographen von besonderem Interesse und Wert sind. Deshalb soll die sich bietende Gelegenheit zur Demonstration einiger weniger Versuchsproben an Hand nachfolgend genannter Kleinbild-Farbdiapositive wahrgenommen werden.

1. Gipfel und Südwestflanke der Dent de Ruth (2236,2). a) Fliegeraufnahme (15. 10. 45); b) Fliegeraufnahme (15. 10. 45); c) Kartenausschnitt aus der Landeskarte 1:50000.

2. Städtische Siedlung, Natur- und Kulturlandschaft. Beispiel: Bellinzona. a) Fliegeraufnahme (4. 10. 45); b) Kartenausschnitt aus Übersichtsplan 1:10000.

3. Voralpine Hangsiedlung in Waldlichtungen. Beispiel: Beatenberg. a) Fliegeraufnahme (17.10.45); b) Kartenausschnitt aus der Landeskarte 1:50000.

4. Voralpine Streusiedlung-Bergbauernhöfe. Beispiel: Simmental-Hofstätten ob Garstatt. a) Fliegeraufnahme (10.10.45); b) Kartenausschnitt aus der Landeskarte $1: 50000$.

1 Referate in der Sektion für Geographie und Kartographie der 127. Jahresversammlung der SNG. in Genf vom 30. August bis 1. September 1947. 